\title{
CiVo: Real-Time Visualization of Social Activities by Cartoonized Twitter
}

\author{
Mitsuru Nakamura, Yosuke Miyazawa, Yoshikazu Kidera, \\ Tsuyoshi Moriyama, and Motoyasu Tamaki \\ Dept. of Manga \& Dept. of Media and Image Technology, Tokyo Polytechnic University, \\ 1583 Iiyama, Atsugi, Kanagawa 243-0297, Japan \\ civo.tpulgmail.com \\ http://civo.sakura.ne.jp/CiVo/CiVo.html
}

\begin{abstract}
We propose a web system called CiVo (City Voice) that is capable of visualizing public conversations on the Internet. It monitors the posting on the Twitter with respect to a set of specific phrases and displays the correspondent movie clips with cartoons on the time line on CiVo when it finds them. It extends the Twitter to a visual social medium that allows people to share what happens in the world at a glance. The main loop of CiVo runs on PHP while monitoring the Twitter using its API and it returns a specific SWF file to the HTTP clients for each GET request. The SWF file always updates its subSWFs reflecting the phrases found on the Twitter.
\end{abstract}

Keywords: Twitter, web services, cartoonization, Action Script, PHP.

\section{Introduction}

Twitter is the most popular social media in present [3][6]. It permits only short postings and reduces lots of efforts that have been required with conventional web pages for making them flawless and up to date. To further enhance the power of Twitter, visualization of the textual postings in pictorial forms is effective and there have been many interesting works [5][4][1][2]. Most of the works visualize the relation between tweets and the tweeters based on their personal profiles. To understand their topics, however, viewers still need to read the texts. We propose a novel visualization that cartoonizes the Twitter. It finds a set of key phrases and expresses their meanings and nuances in cartoons so the viewers can visually and intuitively share the public interest and events at a glance.

\section{How to Cartoonize the Textual Tweets}

People can view the CiVo site by following a special Twitter account "xxCiVoXxx", that follows back the users so that their tweets appear on the time line of "xxCiVoXxx". Fig.1 shows two major modules of the proposed system. One of the modules monitors the time line of "xxCiVoXxx" using Twitter API and searches a specific set of keywords and generates the background component ("background.xml" 
and "weather.xml") and the object component ("objects.xml") of the whole scene in the web page of CiVo. Those XML files hold the hyperlinks to the movie clips (SWFs) correspondent with the keywords that have been tweeted (Fig.1(a)). The other module returns a HTML file "CiVo.html" responding to each HTTP/GET request that embeds a SWF file "CiVo.swf" that internally loads sub-SWFs that have already been specified in the XML files as mentioned above (Fig.1(b)). "CiVo.swf" shows a walking girl on the ground of a small planet that rotates slowly. The ground has no objects until the keywords are tweeted.

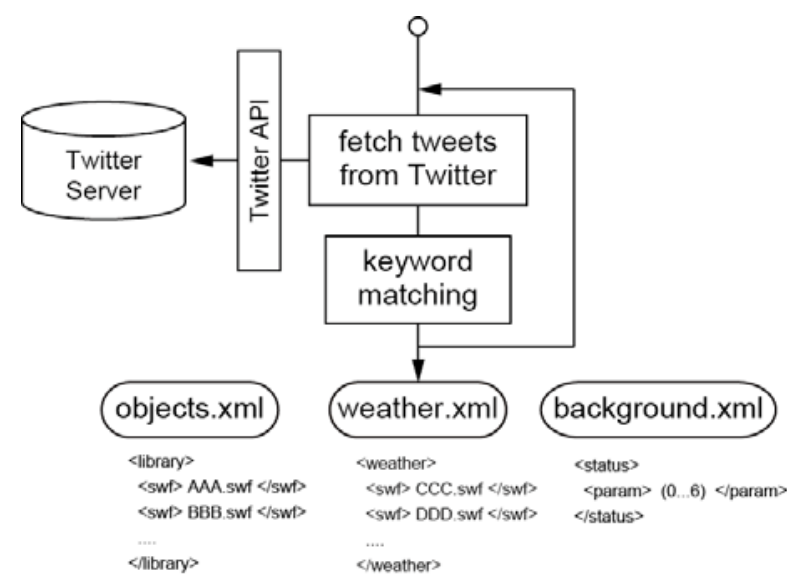

(a) Determination of sub-SWFs to be contained in CiVo web page.

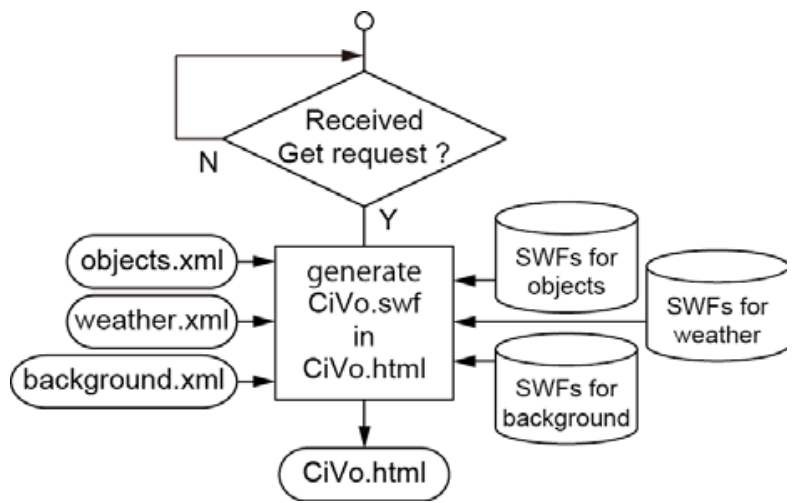

(b) Creation of CiVo page.

Fig. 1. The proposed method of tweet cartoonization

An example set of keywords and their correspondent cartoons that have been currently implemented are shown in Fig.2. Those keywords are currently implemented in Japanese (extensible to any languages). During the execution time, 
the system reloads the pairs of keywords and their correspondent cartoons (SWFs) from a single text file every minute. It allows the service provider to add new sets of keywords and the correspondent cartoons without restarting the system. Fig.3 shows examples of translation from a keyword to the correspondent cartoon.

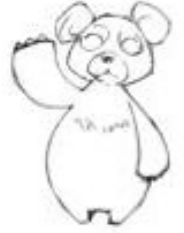

(a) "bear".

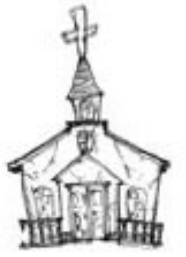

(f) "church".

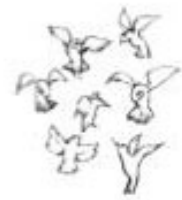

(b) "bird".

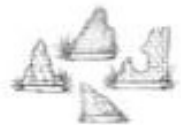

(c) "bricks".

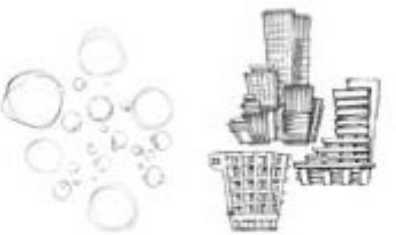

(d) "bubble". (e) "building".
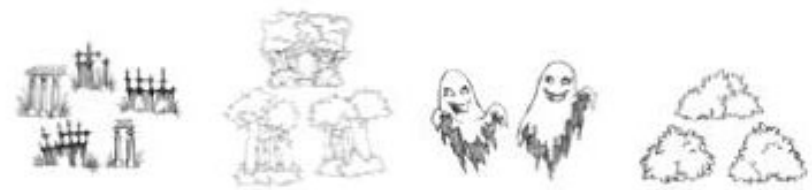

(g) "fence".

(h) "forest".

(i) "ghost".

(j) "grass".
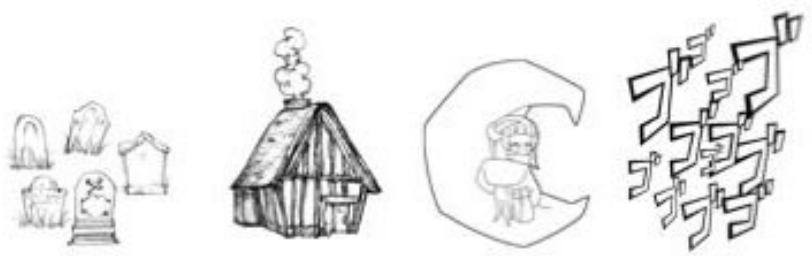

(l) "house".

(m) "moon".

(n) "anger".

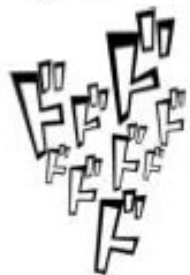

(o) "hurry".

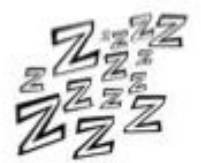

(p) "sleep".

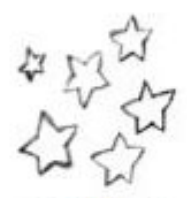

(q) "star".

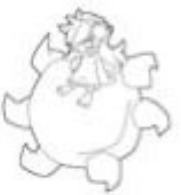

(r) "sun".

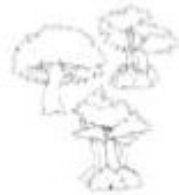

(s) "tree".

Fig. 2. Keywords and the correspondent cartoons 


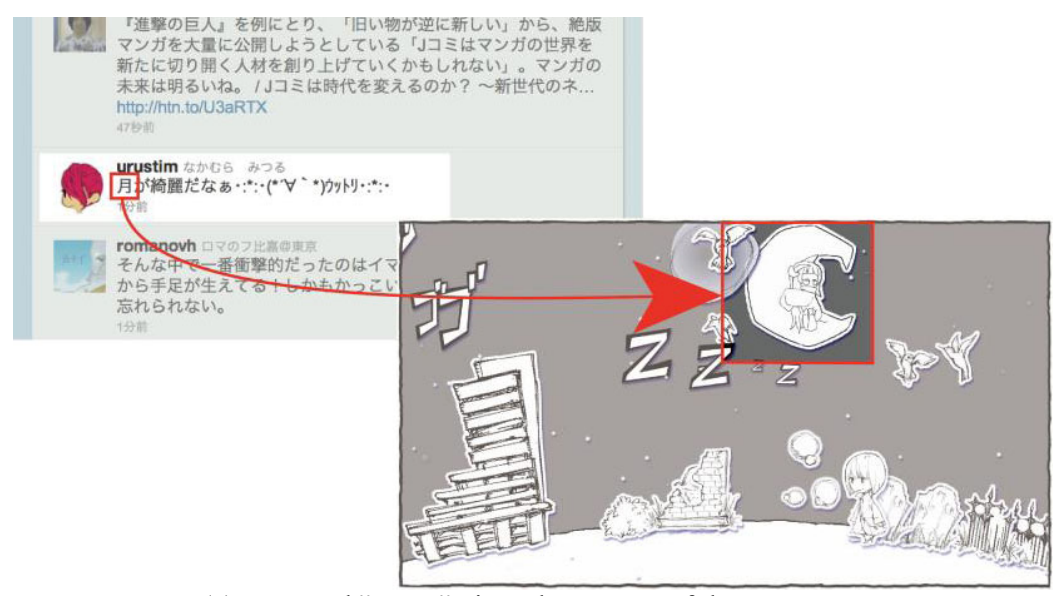

(a) Keyword "moon" gives the cartoon of the moon.

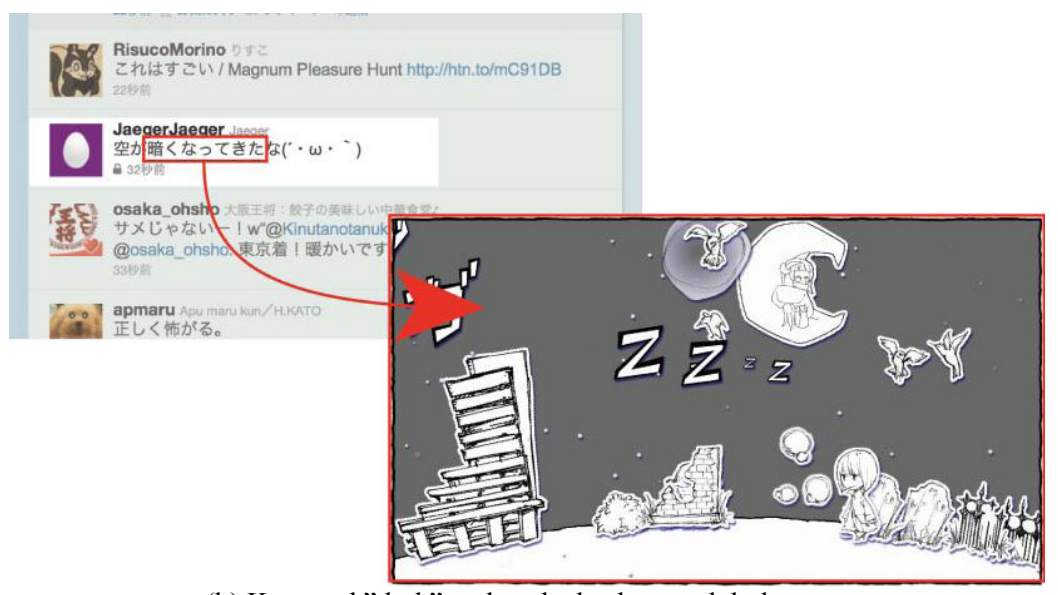

(b) Keyword "dark" makes the background darker.

Fig. 3. Examples of cartoonization by the proposed method

\section{Conclusion}

We proposed a web system called CiVo that translated textual conversations on Twitter in the form of cartoons. It enables the viewer to grasp what people talk about at a glance. Future works include installation to public facilities such as train stations.

Acknowledgments. A part of this work has been supported by the Konica Minolta Image Science Award 2010. 


\section{References}

1. 17 ways to visualize the twitter universe, http://flowingdata.com/2008/03/12/17-ways-tovisualize-the-twitter-universe/

2. Is parade, http://isparade.jp/

3. Twitter, http://twitter.com/

4. Demirbas, M., Bayir, M., Akcora, C., Yilmaz, Y., Ferhatosmanoglu, H.: Crowd-sourced sensing and collaboration using twitter. In: 2010 IEEE International Symposium on a World of Wireless Mobile and Multimedia Networks (WoWMoM), pp. 1-9. IEEE, Los Alamitos (2010)

5. Diakopoulos, N., Naaman, M., Kivran-Swaine, F.: Diamonds in the rough: Social media visual analytics for journalistic inquiry. In: 2010 IEEE Symposium on Visual Analytics Science and Technology (VAST), pp. 115-122. IEEE, Los Alamitos (2010)

6. Java, A., Song, X., Finin, T., Tseng, B.: Why we twitter: understanding microblog-ging usage and communities. In: Proceedings of the 9th WebKDD and 1st SNA-KDD 2007 Workshop on Web Mining and Social Network Analysis, pp. 56-65. ACM, New York (2007) 